Service social

\title{
De la prévisibilité à la diversité des parcours? Le cas de jeunes mères ayant délaissé leurs études
}

\section{Marc Molgat et Caroline Ringuet}

Volume 51, numéro 1, 2004

URI : https://id.erudit.org/iderudit/012712ar

DOI : https://doi.org/10.7202/012712ar

Aller au sommaire du numéro

Éditeur(s)

École de service social de l'Université Laval

ISSN

1708-1734 (numérique)

Découvrir la revue

Citer cet article

Molgat, M. \& Ringuet, C. (2004). De la prévisibilité à la diversité des parcours? Le cas de jeunes mères ayant délaissé leurs études. Service social, 51(1), 60-77. https://doi.org/10.7202/012712ar
Résumé de l'article

Les intervenants sociaux considèrent en général la grossesse à l'adolescence comme un problème important, un point de vue appuyé par de nombreuses recherches qui soulignent les conséquences négatives de la maternité à cet âge de la vie. Le présent article cherche à approfondir cette question en analysant les parcours d'insertion professionnelle de jeunes femmes n'ayant pas terminé leurs études et ayant donné naissance durant l'adolescence ou au début de la vingtaine. Fondés sur une enquête rétrospective conduite par entretiens semi-dirigés, les résultats montrent que ces jeunes mères ne sont pas nécessairement condamnées à l'avance, puisque le soutien familial et les aides à l'insertion permettent à nombre d'entre elles de se stabiliser en emploi. Les aides à l'insertion demeurent cependant défaillantes à plusieurs égards, ce que ne peut résoudre systématiquement le soutien familial. 


\title{
De la prévisibilité à la diversité des parcours? Le cas de jeunes mères ayant délaissé leurs études ${ }^{1}$
}

\author{
Marc MOLGAT \\ Professeur \\ École de service social \\ Université d'Ottawa \\ Caroline RINGUET \\ Travailleuse sociale \\ Conseil des écoles publiques de l'Est de l'Ontario
}

Les intervenants sociaux considèrent en général la grossesse à l'adolescence comme un problème important, un point de vue appuyé par de nombreuses recherches qui soulignent les conséquences négatives de la maternité à cet âge de la vie. Le présent article cherche à approfondir cette question en analysant les parcours d'insertion professionnelle de jeunes femmes n'ayant pas terminé leurs études et ayant donné naissance durant l'adolescence ou au début de la vingtaine. Fondés sur une enquête rétrospective conduite par entretiens semi-dirigés, les résultats montrent que ces jeunes mères ne sont pas nécessairement condamnées à l'avance, puisque le soutien familial et les aides à l'insertion permettent à nombre d'entre elles de se stabiliser en emploi. Les aides à l'insertion demeurent cependant défaillantes à plusieurs égards, ce que ne peut résoudre systématiquement le soutien familial.

Mots clés : grossesse, adolescence, jeunesse, insertion professionnelle, famille, intervention.

Social workers generally consider teenage pregnancy as an important problem, a point of view which is supported by many studies that underline the negative consequences of maternity at this stage of the life course. This article further examines this question through the analysis of labour market trajectories of young women who have not completed their studies and who gave birth during their teenage years or in their early twenties. Based on retrospective semi-directed interviews, the results show that employment outcomes for these young women are not a fore drawn conclusion. Through family support and formal programmes, many manage to obtain stable employment. However, government programmes remain a shaky form of support and the shortcomings of these programmes cannot be systematically made up by help from the family.

Key words: teenage pregnancy, teenagers, youth, employment, family, intervention.

1. Article envoyé pour évaluation le 14 juillet 2004; modifications mineures demandées en août 2005; article accepté en septembre 2005 


\section{INTRODUCTION}

Les intervenants sociaux considèrent en général la grossesse à l'adolescence comme un problème important, un point de vue appuyé par de nombreuses recherches qui soulignent les conséquences négatives de la maternité à cet âge de la vie. Une étude récente menée auprès d'intervenantes et d'adolescentes dans un centre jeunesse au Québec montre, en ce sens, que les intervenantes perçoivent habituellement ces grossesses comme des catastrophes qui ne font qu'ajouter un problème à la liste déjà longue des difficultés auxquelles doivent faire face ces adolescentes (Manseau et Blais, 2003). Les jugements portés sur les adolescentes qui choisissent de mener à terme ces grossesses sont souvent négatifs : ces jeunes femmes ont choisi une stratégie illusoire pour régler leurs problèmes, leurs compétences parentales sont mises en doute, elles sont jugées immatures, voire égoïstes (ibid.). Ces points de vue reposent en grande partie sur les conséquences dites prévisibles de la maternité à l'adolescence, qu'une pléthore d'études nord-américaines s'emploient à relever : risques plus grands de problèmes de santé pour les nouveau-nés et leurs mères (Comité consultatif..., 1999; Combes-Orme, 1993), tendance à ne pas poursuivre ses études (Hockaday et al., 2000), probabilité accrue de connaître des difficultés sur le marché du travail (Charest et Villemure, 2002; Clark, 1999; Côté et Allahar, 1994), faiblesse des revenus (Charest et Villemure, 2002), risques plus élevés de monoparentalité (ibid.), autres ${ }^{2}$.

D'autres chercheurs nuancent cependant ce cliché, mettant en évidence la diversité des situations qui peuvent émerger, au fil des ans, après la mise au monde de l'enfant (Charbonneau, 2003a; Furstenberg, Brooks-Gunn et Morgan, 1987; Hoffman, 1998). Bien que les difficultés soient souvent sérieuses, ces recherches tendent à montrer que les parcours des jeunes femmes ne sont pas prédéterminés par le fait d'avoir accouché avant l'âge de 20 ans. D'autres éléments que celui de l'âge, notamment le recours au soutien familial, l'accès aux aides étatique et communautaire et la présence du conjoint, font diverger les trajectoires, conduisant certaines jeunes femmes vers une plus grande stabilité, d'autres vers des difficultés plus graves encore et d'autres enfin vers un cheminement qui basculera entre stabilité et précarité.

La faiblesse du découpage d'une catégorie d'âge pour traiter de maternité adolescente est par ailleurs mise en évidence par le fait que les âges entre 13 et 19 ans recouvrent des réalités fort différentes. Ainsi, pour ne prendre que quelques exemples, l'obligation scolaire ne s'applique pas aux moins de 16 ans, l'entrée légale dans l'âge adulte est fixée à 18 ans et il n'est pas illégal de former son propre ménage à partir de l'âge de 16 ans. II serait utile, afin d'interroger plus encore le critère de l'âge dans l'étude des conséquences de la grossesse à l'adolescence, d'élargir le spectre et de comparer les jeunes femmes ayant donné naissance avant l'âge de 20 ans avec celles qui ont donné naissance après cet âge, mais avant la fin de leurs études, c'est-à-dire avant le temps aujourd'hui socialement prescrit pour entrer dans la maternité. Nous parlerons alors de maternités précoces plutôt que de grossesses ou de maternités adolescentes,

2. Voir aussi les revues de travaux effectuées par Maynard (1997) et Cournoyer (1995). 
insistant en cela sur les dimensions sociales et construites de cette question ${ }^{3}$, qui ne peut être réduite à un enjeu d'âge ni même de prévention.

II n'en demeure pas moins que les femmes qui deviennent mères à un âge dit précoce, avant ou après 20 ans, sont en quelque sorte catapultées dans la vie adulte par une bifurcation $^{4}$ du parcours qui entraîne un renversement de la logique temporelle des pratiques associées à l'adolescence ou à la jeunesse, dont celles de la fréquentation scolaire et de l'insertion professionnelle. II n'est pas banal de souligner ces deux « bornes » dans le cadre de la maternité précoce, étant donné qu'elles sont aujourd'hui largement perçues comme essentielles à la réussite du passage à la vie adulte, tout en étant de plus en plus franchies vers le milieu ou la fin de la vingtaine. La réussite des jeunes femmes se mesure actuellement par une certaine définition de la performance qui laisse dans l'ombre les parcours des jeunes mères. Selon ce point de vue, il est indispensable d'obtenir d'abord ses diplômes, puis de décrocher l'emploi qui permettra d'atteindre une stabilité financière et d'élargir l'horizon temporel vers des projets qui, enfin, concernent le couple et la famille.

Observant ce discours dans leurs pratiques, les intervenants peuvent être vivement tentés de souligner les conséquences néfastes de la décision de devenir mère, voire de conclure à l'échec du parcours d'insertion professionnelle avant même que celui-ci puisse prendre forme. Mais les jeunes mères sont-elles réellement toutes condamnées à l'avance? La maternité précoce exclut-elle une insertion stable des jeunes femmes dans le marché du travail, les condamnant presque inexorablement à la précarité, au blocage de l'avancement professionnel et à l'impossibilité de se projeter dans l'avenir? Une fois la décision prise de garder le bébé, d'autres parcours sont-ils possibles?

Afin de répondre à ce questionnement, nous proposons dans un premier temps d'examiner quelques données concernant l'insertion professionnelle des jeunes mères ayant délaissé leurs études, en faisant ressortir leur spécificité par rapport aux autres jeunes adultes. Puis, en tentant de nuancer ce portrait et d'en décrire le dynamisme du point de vue des parcours des jeunes mères elles-mêmes, nous analyserons des données issues d'une enquête conduite par entretiens semi-dirigés auprès de jeunes filles ayant délaissé leurs études en 1997.

3. Voir Côté (1997) pour une analyse historique de la contribution des discours en service social et en santé publique à la construction de la « maternité adolescente » au Québec. Aujourd'hui, est considéré comme normal le fait d'avoir un premier enfant après l'adolescence, c'est-à-dire après avoir terminé ses études.

4. Johanne Charbonneau (2003a) définit la maternité adolescente comme un « événement bifurcatif » par rapport aux normes du parcours biographique selon lesquelles devraient se succéder fin de la scolarité, début de l'insertion professionnelle, départ du foyer parental, mise en couple et, enfin, naissance du premier enfant (p. 38-39). 


\section{L'INSERTION PROFESSIONNELLE DES JEUNES MÈRES AYANT DÉLAISSÉ LEURS ÉTUDES.}

Les données existantes au sujet de l'insertion professionnelle des jeunes non diplômés peuvent nourrir le point de vue selon lequel la grossesse précoce conduit presque inévitablement à l'échec.

Il faut bien sûr souligner, en premier lieu, que les jeunes femmes en général sont moins nombreuses que les jeunes hommes à ne pas terminer leurs études secondaires et collégiales dans les délais prescrits ${ }^{5}$ et que les profils de sortie de l'école des garçons et des filles ne sont que légèrement différents ${ }^{6}$. Au secondaire, les jeunes hommes autant que les jeunes femmes invoquent principalement l'incapacité de l'école à les intéresser pour justifier leur départ. Par contre, les hommes soulignent plus que les femmes leur désir de travailler et, les femmes plus que les hommes, l'arrivée et l'éducation d'un enfant. Ainsi, dans certaines situations, la grossesse peut provoquer le départ de l'école, mais elle ne constitue pas pour les femmes le seul facteur en cause.

Du point de vue de l'insertion professionnelle elle-même, d'autres données montrent que, dans l'ensemble, les jeunes qui ne possèdent pas de diplôme secondaire ou collégial ont, par rapport à ceux qui ont obtenu ces diplômes, des taux de chômage plus élevés et des salaires plus bas (Vultur, Trottier et Gauthier, 2002). Ces taux et niveaux de rémunération sont moins favorables encore pour les jeunes femmes, du moins au secondaire. Selon l'Enquête de suivi auprès des sortants, réalisée par sondage à l'échelle du Canada en 1995, les jeunes femmes âgées de 22 à 24 ans qui ont quitté les études secondaires avant l'âge de 18 ans sans avoir obtenu de diplôme connaissent un taux de chômage de 30 \%, alors que ce taux chez les hommes du même âge n'atteint que 17 \% (DRHC, 1998). En outre, selon la même étude, les salaires hebdomadaires moyens de ces femmes sont inférieurs à ceux des hommes, ce qui refléterait un taux d'emploi à temps partiel plus élevé chez les premières ainsi qu'une comptabilisation du salaire excluant les revenus d'emplois autres que ceux rattachés à l'occupation « principale » (ibid. $)^{7}$. Enfin, on remarquera que les femmes non diplômées du secondaire participent moins au marché du travail et reçoivent plus de revenus de l'aide sociale et des allocations familiales (ibid.).

Ces différences dans le parcours d'insertion professionnelle s'expliquent en grande partie par les responsabilités familiales qui incombent plus tôt aux jeunes femmes non

5. Le taux de « décrochage » scolaire au Québec (proportion de la population de 17, 18 ou 19 ans qui ne fréquente pas l'école et qui n'a pas obtenu de diplôme du secondaire), en 2000-2001, est de 18,8\% chez les 19 ans; la différence entre les sexes est importante : 23,9 \% chez les hommes et 13,4 \% chez les femmes (MEQ, 2003; voir le tableau de la fiche 2.6). Au collégial, en 2000-2001, ces taux sont de $31,4 \%$ dans la filière de formation préuniversitaire et de $42,7 \%$ en formation technique. Les taux sont plus élevés chez les hommes que chez les femmes, tant en formation préuniversitaire que technique (MEQ, 2003; voir les tableaux des fiches 3.3 et 3.4.).

6. Voir Conseil supérieur de l'éducation (1996) et Fédération des cégeps (1999).

7. En effet, comme le souligne l'étude, l'écart de salaire entre femmes et hommes non diplômés du secondaire rétrécirait si toutes les sources de revenu étaient calculées, puisque les femmes sont plus nombreuses à cumuler deux ou plusieurs emplois (DRHC, 1998). 
diplômées du secondaire. Après le départ de l'école, la suite des trajectoires des jeunes hommes et femmes se différencie assez nettement du point de vue de l'entrée dans la vie familiale ${ }^{8}$. L'Enquête de suivi auprès des sortants montre en ce sens que, parmi les jeunes femmes âgées de 22 à 24 ans qui ont quitté les études secondaires avant l'âge de 18 ans et qui n'ont pas obtenu de diplôme, seulement $41 \%$ sont encore célibataires (contre $63 \%$ des hommes) et $64 \%$ ont déjà un ou plusieurs enfants à charge. Ce dernier taux est nettement plus élevé que ceux des jeunes hommes qui ont suivi le même parcours (28\%) et des diplômés, qu'il s'agisse d'hommes (7\%) ou de femmes (16 \%) (DRHC, 1998).

Même si elles donnent à penser que l'abandon des études et la maternité précoce peuvent mener à des situations d'emploi et de travail peu enviables pour les jeunes femmes, ces données ne renseignent guère sur la dynamique de leurs parcours d'insertion professionnelle et de leur stabilisation éventuelle. D'une part, elles ne tiennent pas compte des relations et des soutiens dont disposent les jeunes femmes; d'autre part, elles ne suivent pas leurs parcours dans le temps.

II en va ainsi de cette recherche comme de beaucoup d'autres à propos des maternités précoces. Johanne Charbonneau souligne cette lacune et propose de la combler en adoptant, dans ses recherches, une démarche liant parcours de vie et réseau social (2003, p. 36-45). La maternité adolescente est alors considérée à la fois comme un événement «bifurcatif » qui vient modifier le cycle de vie et comme un phénomène social ancré dans des relations de dépendance, d'indépendance et d'interdépendance (ibid.).

Dans la section suivante, nous avons choisi d'épouser ces principes afin d'analyser les parcours d'insertion professionnelle de jeunes mères ayant délaissé des études en 1997, en nous concentrant sur le niveau d'insertion professionnelle de ces jeunes femmes et sur les rôles joués à cet égard par la famille et les mesures d'aide à l'insertion. L'analyse concerne à la fois des jeunes mères ayant donné naissance avant l'âge de 20 ans et d'autres ayant donné naissance plus tard, mais avant l'âge moyen de la première naissance au Québec ${ }^{9}$. Nous relevons, au cours de l'analyse, les distinctions entre les jeunes femmes ayant donné naissance à l'adolescence et celles qui ont eu leur premier enfant plus tard.

\section{MÉTHODOLOGIE DE L'ENQUÊTE}

L'étude présentée s'inscrit dans un projet de recherche plus vaste portant sur l'insertion professionnelle et sur le rapport au travail des jeunes qui ont abandonné le

8. Nous n'avons pas trouvé d'études qui mettent en évidence ces dimensions des parcours des jeunes non diplômés du collégial.

9. Au Québec, en 2003, l'âge moyen des femmes qui donnent naissance pour la première fois est de 27,6 ans (Institut de la statistique du Québec, 2004). 
système d'enseignement secondaire ou collégial en $1996-1997^{10}$, soit quatre à six ans avant l'entrevue. L'objectif principal du projet a consisté à recueillir auprès des jeunes eux-mêmes le récit du chemin parcouru entre la sortie de l'école et le moment de l'entrevue. En outre, devant le peu de connaissances sur ces jeunes, le projet visait à analyser leur rapport au travail, leurs stratégies, la conception qu'ils se font de leur insertion et leurs attentes par rapport aux mesures d'aide à l'insertion et aux initiatives de formation continue. L'échantillon de l'enquête a été constitué selon une méthode non probabiliste à partir de la Banque sur les cheminements scolaires du ministère de l'Éducation du Québec. Le recrutement des sujets s'est effectué pendant près d'une année, sur une base volontaire, auprès de jeunes ayant fréquenté des écoles secondaires et des cégeps dans les communautés urbaines de Montréal (île de Montréal), de Québec et de l'Outaouais. En vue de recueillir les données, 98 entretiens d'environ une quarantaine de minutes ont été réalisés à partir d'un schéma d'entretien semidirigé, auprès d'un nombre relativement égal de jeunes femmes $(n=47)$ et hommes $(n=51)$ ayant délaissé soit le secondaire $(n=47)$, soit le collégial $(n=51)$. En outre, les chercheurs ont utilisé comme matériel de collecte de données une fiche de renseignements sociodémographiques et un calendrier pour faire le suivi des trajectoires depuis l'abandon des études. Le schéma d'entretien regroupait sept thèmes : le cheminement scolaire, la trajectoire professionnelle, le rapport au travail, les représentations de l'insertion professionnelle, les autres dimensions du passage à la vie adulte, les mesures d'aide à l'insertion et l'avenir (représentations et projets). Parmi tous les jeunes interrogés, nous avons retenu pour cet article uniquement les filles qui ont délaissé en 1996-1997 l'école secondaire ou le cégep et qui ont donné naissance à un ou plus d'un enfant $(n=15)$.

Le verbatim des entretiens a été soumis à une analyse de contenu par thème. Nous avons d'abord établi certaines catégories d'analyse, notamment la situation scolaire des répondantes (parcours scolaire, raisons du départ, perspectives sur le retour éventuel aux études), la situation professionnelle (trajectoire, influence de la grossesse sur le travail, perspectives d'avenir), le soutien familial (parcours familial, soutien des parents et du conjoint, conciliation famille, loisirs et amis et projets d'avenir pour la famille) et le soutien externe (aide gouvernementale ou soutien venant de divers organismes). Par la suite, nous avons regroupé le contenu des entrevues sous différents thèmes pour leur donner un sens dans le cadre de cet article.

\section{DESCRIPTION DES JEUNES MÈRES INTERVIEWEÉES}

L'échantillon est formé de quinze jeunes femmes âgées de 21 à 27 ans, dont neuf ont entre 21 et 24 ans et six, entre 25 et 27 ans. Seules deux femmes sont en situation de monoparentalité au moment des entretiens, tandis que treize autres vivent en couple. En ce qui concerne le plus haut niveau de scolarité atteint, l'échantillon est composé de neuf jeunes femmes qui n'ont pas obtenu leur diplôme d'études secondaires (DES).

10. Le projet a été mené par Madeleine Gauthier (responsable du projet), Jacques Hamel, Marc Molgat, Claude Trottier et Mircea Vultur et subventionné par le Fonds québécois sur la recherche et la culture (FQRSC), dans le cadre du programme de l'Action concertée sur le travail en mutation. 
Chez ces femmes, le niveau de scolarité varie entre la première et la quatrième année du secondaire, et une répondante a obtenu une attestation d'études collégiales. Parmi celles qui ont obtenu un DES (6), une seule n'a pas terminé au moins une année d'études postsecondaires. Les autres ont interrompu leurs études collégiales entre la première et la quatrième année suivant leur inscription à ce niveau d'études.

Certaines ont eu un enfant durant leur adolescence (8) et d'autres (6) lorsqu'elles étaient plus âgées ${ }^{11}$. De plus, la plupart des répondantes de notre étude (11/15) n'ont pas abandonné l'école parce qu'elles étaient enceintes. La grossesse n'explique donc pas toujours l'abandon du parcours scolaire, la grande majorité ayant quitté les études avant d'être enceintes. Pour celles qui sont enceintes au moment de quitter les études, le parcours scolaire antérieur est toujours marqué par des difficultés et des échecs.

Du point de vue de leur propre famille, huit jeunes femmes ont donné naissance à un seul enfant, alors que cinq ont eu deux enfants. De plus, deux répondantes sont enceintes pour une seconde fois au moment de l'entrevue.

En ce qui a trait à leur situation professionnelle, onze sont actives sur le marché du travail au moment des entretiens, deux sont en congé de maternité et perçoivent des prestations d'assurance-emploi, tandis que deux bénéficient de l'aide sociale (dont une occupe également un emploi à temps partiel). La plupart (11/15) ont un emploi à temps plein ou sont sur le point d'en obtenir un, alors qu'une seule occupe un emploi à temps partiel; une autre travaille à temps plein durant l'été et à temps partiel pendant l'hiver, tandis que la dernière travaille sur appe ${ }^{12}$. La fourchette des revenus hebdomadaires est assez large, ceux-ci variant entre 212 \$ et 2400 \$. Seulement trois participantes ont un salaire de $300 \$$ et moins par semaine, alors que huit gagnent entre $301 \$$ et 400 \$, deux entre 401 \$ et 500 \$ et une entre 601 \$ et 700 \$. Enfin, une jeune femme a un revenu de plus de $2000 \$$ par semaine.

En ce qui concerne la scolarité des parents, les pères de sept répondantes n'ont pas obtenu de diplôme d'études secondaires (DES). Parmi eux, trois n'ont atteint que le niveau primaire et quatre ont délaissé leurs études au secondaire. Parmi ceux qui ont obtenu un DES (5/15), deux n'ont pas poussé plus loin leurs études, deux ont fait des études collégiales et un, des études universitaires. Enfin, deux pères ont obtenu un diplôme d'études professionnelles $(D E P)^{13}$. Quant aux mères des répondantes, six d'entre elles n'ont pas obtenu un DES. Parmi ces dernières, deux ont un niveau de scolarité ne dépassant pas le primaire, trois ont mis fin en cours de route à leurs études secondaires et une a obtenu une équivalence d'études secondaires. L'une des mères possède un DEP. Enfin, quatre mères ont obtenu leur DES et une, un diplôme universitaire . $^{14}$.

11. Les données précises à ce sujet ne sont pas disponibles pour une des répondantes, mais selon les informations recueillies elle a donné naissance avant l'âge moyen des femmes à la première naissance au Québec.

12. Nous ne disposons pas de données à ce sujet pour une des répondantes.

13. Les données au sujet de la scolarité du père ne sont pas disponibles pour une des répondantes.

14. Les données sur la scolarité de la mère ne sont pas disponibles pour trois répondantes. 


\section{LES PARCOURS D'INSERTION PROFESSIONNELLE, LE SOUTIEN FAMILIAL ET LES AIDES À L'INSERTION}

Les données statistiques et quantitatives que nous avons rapportées sur l'insertion professionnelle des jeunes qui n'ont pas de diplôme d'études secondaires ou collégiales révèlent que les difficultés d'insertion dans le marché du travail peuvent être importantes, et plus encore pour les jeunes femmes. De surcroît, les salaires relativement faibles de plusieurs jeunes mères interviewées dans le cadre du projet de recherche peuvent présager d'une précarité assez grande. Cependant, les parcours sont plus dynamiques et plus divers qu'il n'y paraît au premier abord. Ainsi, en évaluant le niveau d'insertion professionnelle en termes de stabilité d'emploi et de satisfaction au travail des jeunes mères, nous constatons que plusieurs de celles-ci réussissent à tirer leur épingle du jeu. Nous examinerons, dans les pages qui suivent, par quels moyens la stabilité est obtenue ou, au contraire, ce qui la freine, en étudiant plus spécifiquement les logiques du soutien familial et des aides à l'insertion dans ce processus. Nous conclurons en dégageant de l'analyse quelques perspectives pour l'intervention.

II existe diverses typologies pour catégoriser les trajectoires professionnelles des jeunes. L'analyse de l'ensemble des entretiens réalisés pour le projet de recherche a mené à la construction d'une typologie qui s'inspire de celle proposée par Demazière et Dubar dans une étude sur des jeunes « de bas niveau scolaire » en France (1994, p. 80$)^{15}$. La typologie permet de circonscrire quatre types de trajectoires selon le niveau de stabilité de l'insertion professionnelle et la présence sur le marché du travail : a) les jeunes qui se sont stabilisés sur le marché du travail; b) les jeunes qui sont en voie de se stabiliser sur le marché du travail; c) les jeunes qui sont en situation précaire; d) les jeunes qui sont en marge du marché du travail. Nous avons inscrit les récits des répondantes dans les catégories de cette typologie afin de préciser leur niveau d'insertion professionnelle. Puis, pour chacune des catégories, nous avons décrit la satisfaction des jeunes mères à l'égard de leur emploi. La satisfaction en emploi est évaluée, à la suite des travaux de Paugam (2000), selon l'épanouissement personnel qu'il accorde, les avantages matériaux qu'il procure ainsi que l'ambiance et les relations humaines dans le milieu du travail. La combinaison de ces deux dimensions (niveau d'insertion et satisfaction au travail) permet de qualifier plus globalement l'insertion professionnelle des jeunes mères. En parallèle, et pour tenter de rattacher les parcours des répondantes au tissu relationnel et d'aide dans lequel ils se déploient, nous cernons la logique des soutiens familiaux et des mesures d'aide dans les parcours d'insertion professionnelle en répondant à deux questions : 1) À quels moments interviennent les soutiens familiaux et les mesures d'aide à l'insertion? 2) Contribuentils à la stabilisation des parcours?

15. Voir le rapport de recherche pour une présentation détaillée de cette typologie et une analyse de l'ensemble des entretiens (Gauthier et al., 2004). 


\section{LES JEUNES MÈRES STABILISÉES}

Une surprise de taille nous attendait dès le classement des parcours dans la première catégorie : la plupart des trajectoires des jeunes mères (10/15) les rattachait au groupe de jeunes qui se sont stabilisés sur le marché du travail. II s'agit ici de jeunes qui sont en mesure de subvenir à leurs besoins, de maintenir leur autonomie financière et de former des projets de vie réalisables ${ }^{16}$. La stabilité sur le marché du travail des jeunes interviewées a été atteinte parce que celles-ci occupaient un même emploi depuis plus de deux ans (8) ou avaient occupé plusieurs emplois dans un même domaine sans avoir connu de période de chômage pendant plus de deux ans (1) ou encore occupaient depuis plus de deux ans un emploi en alternant, à leur convenance, entre travail à temps plein et travail à temps partiel (1). L'examen des caractéristiques de ces répondantes montre que presque toutes les jeunes mères ayant délaissé des études collégiales (4/5) se retrouvent dans cette catégorie. Mais on y trouve également la plupart des jeunes femmes ayant donné naissance durant leur adolescence (5/8).

Presque toutes ces jeunes femmes expriment de la satisfaction à l'égard de leur travail, seules deux répondantes se déclarant insatisfaites. La première, qui travaille à la chaîne dans une usine de confection de vêtements, estime que son emploi est peu valorisant et elle évalue défavorablement son salaire et ses relations de travail avec ses collègues (qui parlent une autre langue que le français et lui adressent rarement la parole). Elle espère trouver bientôt un autre emploi. La seconde est plutôt satisfaite de son salaire et du milieu de travail, mais juge que son emploi ne lui donne pas l'occasion de s'accomplir.

En général, ces jeunes mères ont bénéficié, plus que les autres interviewées, de soutiens familiaux et d'aides à l'insertion professionnelle. Chacune d'entre elles a reçu, à une ou plusieurs reprises durant les quatre à six années depuis sa sortie de l'école ou de cégep, des soutiens de sa famille ou de son conjoint. Dans plusieurs cas (8), des mesures d'aides à l'insertion se sont ajoutées.

Les soutiens de la famille ou du conjoint interviennent dans plusieurs cas immédiatement après la sortie de l'école ou du cégep: soit que les parents ou la famille acceptent que l'interviewée habite au domicile familial en attendant d'avoir davantage de stabilité, soit que l'aménagement avec le conjoint signale le début d'un processus de construction commune de l'insertion sociale et professionnelle. Dans le premier cas de figure, les jeunes femmes vont cohabiter avec leurs parents et parfois leurs beauxparents pour des périodes comprises entre six mois et deux ans. Pendant cette période, toutes occuperont un emploi ou retourneront aux études, et quelques-unes y vivront une période de chômage et même une grossesse. Une interviewée y demeurera jusqu'à deux mois après l'accouchement, moment où elle partira vivre avec son

16. Les projets de vie sont considérés comme réalisables lorsqu'un jeune a acquis une autonomie suffisante pour avoir des projets et pouvoir les mettre en œuvre. Ces projets peuvent être d'ordre personnel, comme l'achat d'une maison, la naissance d'un enfant ou encore un voyage. Ils peuvent aussi être d'ordre professionnel, comme le passage à un statut d'emploi amélioré dans son entreprise, le démarrage d'une entreprise, du perfectionnement ou un retour aux études (Gauthier et al., 2004). 
conjoint. Dans le second cas, les jeunes femmes déménagent immédiatement après avoir délaissé leurs études pour s'installer en couple. S'amorce alors pour ces jeunes femmes une période où la conciliation de la vie familiale et de la vie professionnelle est prise en compte pour les deux conjoints. L'arrivée des enfants n'empêche donc pas l'insertion professionnelle de ces femmes, cette insertion étant déjà bien amorcée, voire stabilisée au moment de la naissance du premier enfant. Le soutien du conjoint, dans ces cas, est présent tout au long de la trajectoire des jeunes mères. Dans trois cas, l'insertion et la stabilisation professionnelle de ces dernières sont même synonyme de leur intégration au sein de l'entreprise fondée par le couple.

Les soutiens de la famille ou du conjoint peuvent aussi intervenir à au moins deux autres moments des parcours des jeunes mères dont l'insertion professionnelle s'est stabilisée: Iorsque le jeune couple vit une période de pauvreté et lorsque les mères occupent un emploi à horaire irrégulier. L'aide matérielle des parents du jeune couple peut ainsi se traduire par l'aménagement et la décoration du logement, l'achat de nourriture et du nécessaire pour le bébé, etc. Et lorsque les jeunes mères occupent des emplois où elles doivent travailler sur appel, les soirs ou les fins de semaine, des membres de la famille, comme le conjoint, le père, la belle-sœur, la belle-mère, se prêtent volontiers à la garde des enfants et permettent ainsi aux jeunes femmes de maintenir une certaine stabilité d'emploi. Ainsi, Geneviève ${ }^{17}$, dont l'emploi est sur appel « sept jours sur sept, vingt-quatre heures sur vingt-quatre », doit compter sur «sa belle-mère [qui est] proche » pour la garde de sa fille lorsqu'elle et son conjoint ne peuvent pas s'en occuper.

Ce dernier exemple vient souligner jusqu'à quel point les services de garde paraissent essentiels pour assurer la stabilité de l'insertion professionnelle des jeunes femmes dont il est ici question. Si, dans le cas que nous venons de citer, la couverture du service de garde est incomplète au regard de l'horaire de travail, il n'en demeure pas moins que les autres interviewées « stabilisées » ont recours à une place en garderie subventionnée au moment où elles doivent se trouver un emploi ou effectuer un retour aux études, ou encore elles envoient leur ou leurs enfants à la garderie lorsqu'elles sont sur le marché du travail ${ }^{18}$. Dans le cas des jeunes mères qui ont des horaires irréguliers, la complémentarité de cette forme de service avec le soutien de la famille paraît incontournable pour assurer la stabilité de l'insertion professionnelle.

Les prestations de l'assurance-emploi dans le cadre d'un congé de maternité constituent une autre forme d'aide à l'insertion professionnelle à laquelle ont souvent recours ces jeunes mères. Seules deux interviewées n'en font pas usage, puisqu'elles ne travaillent pas au moment de leur grossesse. Toutes les autres ont pu y avoir accès pour des périodes allant jusqu'à un an. Cependant, il faut noter que cette forme d'aide - qui permet aux parents de s'occuper de leurs enfants en bas âge tout en conservant leur lien d'emploi - ne paraît accessible que dans la mesure où les interviewées vivent avec un conjoint qui travaille au moment où débute le congé de maternité. En effet,

17. Les noms utilisés sont fictifs.

18. Il est à noter qu'il est impossible dans plusieurs cas de déterminer si les places obtenues en garderie sont subventionnées ou non. Une question à cet égard ne figurait pas dans le schéma d'entretien. 
sauf exception, il aurait été impossible pour ces jeunes mères de faire vivre leur famille avec un salaire amputé de $45 \%$, comme le prévoyait au moment de l'enquête le programme de congé de maternité de l'assurance-emploi.

Enfin, notons que ces jeunes mères ont occasionnellement eu recours à d'autres mesures d'aide à l'insertion professionnelle. II s'agit des guichets d'emploi (jugés très utiles, dans deux cas, pour trouver un premier ou un deuxième emploi) et les carrefours jeunesse-emploi (dans quatre cas, dont trois pour lancer une entreprise et un pour s'orienter en emploi).

\section{LES JEUNES MÈRES EN VOIE DE SE STABILISER}

La deuxième catégorie regroupe les jeunes mères qui sont en voie de se stabiliser sur le marché du travail (4/15). S'y retrouvent les répondantes qui, comme dans le groupe précédent, sont en mesure de subvenir à leurs propres besoins, de maintenir leur autonomie financière et de former des projets de vie réalisables. Ces femmes, cependant, ont encore à consolider leur processus de stabilisation, car elles occupent depuis moins longtemps l'emploi occupé au moment de l'entretien, soit parce qu'elles sont retournées aux études afin d'obtenir les compétences requises dans un domaine qui les intéresse (2), soit parce qu'elles viennent d'obtenir un emploi permanent (1), soit encore parce qu'elles préparent concrètement une transition d'un emploi stable vers un autre emploi (1). Dans ce groupe de parcours, on retrouve deux femmes ayant donné naissance durant leur adolescence, deux mères monoparentales (dont une a eu son premier enfant avant l'âge de 20 ans) et une seule qui a quitté des études collégiales avant d'avoir terminé.

Une seule de ces jeunes mères est complètement satisfaite de son emploi. II s'agit de Mélanie qui, après un congé de maternité, est allée suivre un cours de massothérapeute. Aujourd'hui employée dans le domaine, mais depuis un an seulement, elle se retrouve en retrait préventif. Elle trouve plus difficile cette absence du travail que celle qu'elle a vécue lorsqu'elle était en congé parental pour son premier enfant. Elle occupait alors un emploi qu'elle jugeait peu valorisant dans le secteur de la vente au

détail. Pour deux autres interviewées, les insatisfactions touchent les relations dans le milieu de travail et la possibilité de trouver une valorisation à leur emploi. L'une d'elles se dit pourtant satisfaite de son salaire et du domaine dans lequel elle travaille, alors que l'autre estime qu'elle a «plafonné » sur le plan du salaire et des possibilités d'avancement.

Bien que l'accès aux mesures d'aide à l'insertion soit globalement favorable à l'insertion professionnelle de ces interviewées, il s'avère plus problématique pour ces femmes que pour celles s'étant stabilisées en emploi. De plus, les soutiens familiaux ne sont pas davantage présents pour ces jeunes mères.

Sauf exception, les répondantes en voie de se stabiliser en emploi ont eu recours à la cohabitation ou à la recohabitation avec leurs parents à des périodes difficiles de leurs parcours : pour deux d'entre elles, la cohabitation fait suite au départ de l'école et dure plus d'un an. Cette période couvre l'obtention d'un premier emploi à temps plein et, 
pour l'une de ces deux femmes, la grossesse ${ }^{19}$ et une période d'un mois après la naissance. Deux interviewées, dont l'une a prolongé la cohabitation après avoir abandonné ses études, sont retournées vivre chez leurs parents à la suite d'une séparation ou du décès du conjoint. Au moment de ces recohabitations, les deux jeunes femmes ont des enfants à charge et doivent donc rapidement trouver un logement plus convenable pour leur famille.

L'une des deux jeunes femmes, Cindy, trouve alors un emploi grâce à l'intervention de son père. Elle va occuper cet emploi - où elle prépare des aliments pour la section « prêt-à-manger » d'une épicerie - pendant près de cinq ans avant d'exprimer son « ras-le-bol » face au salaire qui n'augmente plus et aux horaires de travail irréguliers et peu compatibles avec ses responsabilités familiales. Heureusement, sa mère est là pour s'occuper de sa fille quand elle travaille le soir et rentre après minuit. Cindy cherche, au moment de l'entretien, à effectuer une transition vers un autre type d'emploi en retournant d'abord aux études à plein temps. Elle constate cependant que le centre local d'emploi offre peu de solutions adaptées à sa situation et à ses besoins. En effet, elle apprend que, si elle quitte son emploi de son propre chef, les règlements de l'assurance-emploi lui interdisent d'accéder aux prestations. Elle se voit donc obligée de choisir le moindre de deux maux : étudier à temps plein tout en étant inscrite à l'aide sociale (une option qui lui sourit peu) ou étudier à temps partiel tout en continuant à travailler. Elle choisit finalement l'option qu'elle juge « la moins pire » et, au moment de l'entretien, elle s'est inscrite à un cours offert en soirée. Cindy trouve sa situation malheureuse, car elle estime que plusieurs années encore devront s'écouler avant qu'elle puisse obtenir son DES. Tout en regrettant de ne pas avoir terminé ses études lorsqu'elle était plus jeune, elle dénonce vigoureusement les rigidités des mesures d'aide : «Mais, c'est ça, je trouve ça con la société. Quand tu veux vraiment retourner à l'école, il n'y a pas moyen de t'aider, tu sais. »

L'autre interviewée, Lisa, finit par s'inscrire (à contrecœur) à l'aide sociale afin de compléter au collégial la formation technique en aide juridique qu'elle a entreprise après avoir quitté à nouveau le domicile de ses parents. Pendant cette période, elle se voit obligée de travailler une journée par semaine dans un dépanneur pour augmenter ses revenus, les prestations d'aide sociale ne lui permettant pas de boucler le budget familial tout en payant les coûts associés à ses études. Ainsi, non seulement elle doit s'occuper de ses deux enfants, les envoyer à la garderie et étudier, mais il lui faut en plus travailler un jour par semaine pour ne pas manquer d'argent. Tout comme Cindy, Lisa évalue assez sévèrement cette aide à l'insertion professionnelle, affirmant que les règlements de l'aide sociale l'empêchaient d'obtenir un diplôme et l'orientaient plutôt vers une attestation d'études, voie dans laquelle elle finit par s'engager plus ou moins contre son gré. De plus, elle critique la quantité de «paperasse » à remplir. Elle avoue même avoir trouvé l'expérience de l'aide sociale tellement décourageante qu'elle a presque décidé d'abandonner sa formation.

19. Il ne s'agit pas d'une grossesse au moment de l'adolescence. 
Il faut enfin souligner le cas de Karine qui, au moment de l'entretien, est sur le point de commencer un nouvel emploi permanent et à temps plein comme gérante d'un club vidéo. Elle n'a bénéficié, au cours de sa trajectoire professionnelle, que de la présence de son conjoint, ce qui lui a permis de prendre des congés de maternité à l'occasion de la naissance de leurs deux enfants. L'emploi qu'elle occupait alors ne la suit pas quand elle se voit obligée d'aller vivre plus près de sa famille pour s'occuper de son frère handicapé. Elle doit alors s'inscrire à l'aide sociale et chercher du travail, ce qui n'est pas une mince tâche, puisque, pour accepter un emploi à temps plein, elle doit trouver une place subventionnée en garderie. Dans le cas contraire, une part trop grande de ses revenus devra être consacrée à payer les frais de garde. C'est d'ailleurs l'obtention de cette place qui lui permettra de retourner au travail quelques semaines après l'entretien.

\section{LES JEUNES MÈRES EN MARGE}

Le dernier type de trajectoire que l'on retrouve parmi les répondantes ${ }^{20}$ est celui des jeunes qui sont en marge du marché du travail. Dans ces trajectoires, on trouve des jeunes qui sont sans emploi au moment de l'entretien, qui reçoivent pour la plupart des prestations de la sécurité du revenu ou qui ont un emploi à temps partiel qui ne leur permettra pas de subvenir à leurs besoins pendant les prochains mois. Ces jeunes ont des projets de vie qu'ils pourront éventuellement réaliser plus tard, mais ils auront de la difficulté à les mettre en œuvre avant un certain temps ou sans une aide extérieure. Ce type ne compte qu'une seule jeune mère, qui a donné naissance durant l'adolescence et a vécu de nombreux épisodes de violence conjugale. Martine, âgée de 21 ans, est la plus jeune des répondantes. Elle reçoit depuis plusieurs années des prestations de la sécurité du revenu et travaille à temps partiel comme femme de ménage dans un hôtel, un emploi dont elle se déclare peu satisfaite. Elle a obtenu son premier travail dans ce domaine grâce à sa mère qui a, elle aussi, occupé le même type d'emploi. Outre cet appui, elle a reçu peu de soutien de sa famille depuis qu'elle a quitté il y a plusieurs années le domicile parental (au moment où elle était enceinte). Elle y est certes retournée à quelques reprises lorsqu'elle fuyait la violence de son conjoint, mais ces retours ont toujours été brefs, tout comme l'ont été ses séjours dans des maisons d'hébergement pour femmes victimes de violence. Aujourd'hui, débarrassée de ce conjoint, elle vit avec un autre homme qu'elle considère comme " un protecteur ». Sa fille de 4 ans ne fréquente pas la garderie et Martine refuse de l'y envoyer, préférant s'occuper seule de son éducation. En attendant que sa fille soit d'âge scolaire et que les choses se normalisent dans sa propre vie, Martine continuera à travailler à temps partiel et à recevoir des prestations d'aide sociale. Dans ce cas, ni le soutien de la famille ni les mesures d'aide à l'insertion ne peuvent réellement favoriser à court terme la stabilisation de son parcours d'insertion professionnelle. Martine estime en effet

20. Il est à noter qu'aucune répondante ne peut être catégorisée dans le troisième type de trajectoire, qui correspond aux jeunes en situation d'insertion professionnelle précaire, c'est-à-dire des jeunes qui ne sont pas parvenus à se stabiliser sur le marché du travail. Les jeunes dans cette catégorie ont de la difficulté à subvenir entièrement à leurs besoins, à maintenir leur autonomie financière et à former des projets de vie réalisables, du moins à court terme. Voir Gauthier et al. (2004) pour des précisions au sujet de la définition de ce type. 
qu'avant de pouvoir envisager ainsi la vie elle doit d'abord assurer sa propre sécurité et la stabilité de sa vie familiale.

\section{CONCLUSION}

L'analyse montre que, contrairement à certaines idées reçues, ni la grossesse à l'adolescence ni les maternités précoces n'excluent la possibilité de stabilisation de l'insertion professionnelle des jeunes femmes ${ }^{21}$. Cet événement «bifurcatif », selon le contexte dans lequel il se déroule, a donné lieu à des entrées dans la vie adulte traditionnelles, mais plus rapides, où ne fait défaut que l'absence du diplôme d'études. Cet événement, qui paraît « incongru » - selon l'expression de Le Van (1998) - au regard de la succession instituée des âges de la vie, finit par produire nombre de situations qui se distinguent finalement peu de celles d'autres jeunes femmes faiblement scolarisées. Le premier type présente en ce sens des cas où la stabilité de l'insertion professionnelle est sans doute plus rapidement atteinte que s'il n'y avait pas eu d'enfants. La présence des enfants semble en effet obliger certains jeunes couples à assurer le plus rapidement possible une stabilité financière au ménage. II n'en demeure pas moins que ces jeunes femmes paraissent plus vulnérables que les jeunes sans diplôme n'ayant pas encore d'enfants. Avec des enfants en bas âge, ces jeunes mères peuvent plus difficilement se permettre, tant financièrement que du point de vue du temps dont elles disposent, de retourner sur les bancs d'école pour obtenir un diplôme ou une formation qualifiante. Et sans diplôme il leur serait plus pénible de trouver un autre travail en cas de perte d'emploi.

Les soutiens familiaux et la vie de couple paraissent donc essentiels à la stabilité de l'insertion professionnelle et à des revenus adéquats dans le contexte des maternités précoces des interviewées. La vie à deux permet effectivement de pallier l'insuffisance des revenus associée à des bas niveaux de salaire ${ }^{22}$ et de combler, pour un temps, l'absence de revenus en cas de perte d'emploi. Cette solidarité du couple et les soutiens familiaux sont d'autant plus essentiels que les mesures d'aide répondent de manière parcellaire et parfois inefficace aux difficultés qu'éprouvent les jeunes femmes peu scolarisées ayant donné naissance avant les temps socialement prescrits. Ainsi, s'il existe des places subventionnées en garderie, l'emplacement des garderies et les lacunes dans l'arrimage avec les écoles ou les lieux de formation constituent un pro-

21. Les cas retenus dans le cadre de cette démarche présentent peu de problèmes sévères et durables, ce qui peut surprendre par rapport aux enquêtes qui cherchent à souligner le drame de la maternité précoce. II faut rappeler à cet égard que rares étaient les sujets de l'enquête qui avaient moins de 16 ans au moment de quitter l'école en 1997, ce qui excluait d'emblée des jeunes femmes qui auraient pu donner naissance avant l'âge de 16 ans et qui, éventuellement, auraient pu présenter des trajectoires plus problématiques. Aussi, on ne sait pas pourquoi autant de jeunes mères interviewées vivent en couple, une situation qui ne se retrouve pas dans d'autres enquêtes rétrospectives (Charbonneau, 2003a) ou de suivi (Allen et Bourke Dowling, 1998; Furstenberg et al., 1987), mais qui a généralement un effet positif sur la stabilité financière du ménage. Enfin, on peut se demander si des jeunes mères dans des situations plus précaires ont décliné l'offre de participer à la recherche parce qu'elles n'en avaient pas le temps ou bien ne souhaitaient pas faire état de leurs parcours difficiles.

22. Furstenberg et al. en arrivent aussi à cette conclusion, affirmant que les unions stables rapportent d'importants « dividendes » en matière du statut économique du couple (1987, p. 75). 
blème de taille pour celles qui souhaiteraient poursuivre leurs études (Roy et Charest, 2002); de surcroît, les places ne sont pas aisément accessibles dans toutes les régions du Québec. De plus, les horaires des garderies coïncident rarement avec des horaires de travail irréguliers. Enfin, il faut souligner que le choix de bénéficier des prestations associées au congé parental est essentiellement réservé aux jeunes femmes qui vivent en couple. II serait effectivement impossible pour les interviewées de soutenir seules une famille avec des prestations de $45 \%$ inférieures à leurs salaires déjà modestes.

II faut ajouter à ces observations que les entretiens analysés ne mettent pas en évidence une logique de complémentarité selon laquelle les soutiens familiaux comblent systématiquement les brèches dans les mesures d'aide à l'insertion. La « complémentarité »-qui semble en fait une sorte de réponse familiale aux demandes de flexibilité de la main-d'œuvre -, lorsqu'elle existe, concerne surtout la garde des enfants assumée par les parents ou les beaux-parents et parfois par d'autres membres de la famille. II est important de rappeler à cet égard que la possibilité de recourir aux soutiens familiaux et le niveau de ces soutiens sont très souvent liés à la qualité des relations entre les jeunes et leurs parents, ainsi que des ressources financières dont disposent ces derniers (Molgat et Maunaye, 2003). Dans le cas des interviewées, bien que les relations avec les parents paraissent généralement bonnes, ces derniers sont nombreux à occuper des emplois faiblement ou moyennement rémunérés et quelquesuns reçoivent des prestations d'aide sociale. II faut aussi souligner que le soutien parental est souvent moindre après la naissance des enfants, puisque les jeunes mères sentent qu'elles doivent accéder rapidement à leur autonomie résidentielle si la grossesse est vécue au sein du domicile parental (Charbonneau, 2003b).

II semble essentiel de bien saisir la spécificité de la conciliation entre responsabilités familiales et insertion professionnelle pour ces jeunes mères et les difficultés que cette conciliation induit lorsqu'il est question d'envisager un retour aux études. Pour plusieurs d'entre elles, ce retour ne semble pas pouvoir se réaliser sans que la stabilité financière de leur ménage soit mise en péril. En ce sens, les mesures actuelles d'aide à l'insertion professionnelle répondent bien mal au désir de scolarisation de ces jeunes femmes, et cela, malgré les vœux de formation et de scolarisation livrés par les gouvernements en vue d'encourager l'ajustement des institutions et des individus à la « société du savoir ».

Événements hors normes, les grossesses et les maternités «précoces » forment en creux un puissant interrogateur de la société, des définitions de la jeunesse qu'elle se donne et des institutions qu'elle crée pour répondre à celles et ceux qui se trouvent en marge des sentiers battus. L'analyse présentée montre que ces événements, bien qu'ils fassent rétrécir l'éventail des choix en matière d'insertion professionnelle, ne produisent pas inéluctablement des échecs et peuvent constituer une étape somme toute assez normale dans le parcours d'une vie. À la lumière de l'analyse présentée, il semblerait que la stabilisation ou la déstabilisation des parcours des répondantes dépend, en grande partie, de la manière dont la société réagit à ces événements. II y a ainsi, au-delà des programmes de prévention actuels qui s'inquiètent plus des compé- 
tences parentales des jeunes mères que de leurs conditions d'existence, des actions qui peuvent orienter les parcours après la naissance et auxquelles le monde de l'intervention pourrait contribuer. Bien que des caractéristiques personnelles telles que la détermination peuvent concourir à la stabilisation des parcours, elles ne sont jamais les causes uniques du succès ou des difficultés: les ressources disponibles et les règles sociales élargissent ou limitent le rayon des choix et les capacités d'action. Sur cette voie, la recherche nous semble devoir s'intéresser à la fois aux jeunes mères elles-mêmes et aux soutiens qui leur sont (ou ne sont pas) accordés. II faut donc, d'une part, continuer de s'attarder à la construction de leurs parcours en prêtant une oreille attentive à ce qu'elles souhaitent ou, en rétrospective, à ce qu'elles auraient souhaité en vue de la stabilisation de leur insertion sociale et professionnelle; d'autre part, il faut remettre au centre des préoccupations la question de l'accès aux formes de soutien qui peuvent élargir les possibilités de formation, d'emploi et de conciliation famille-travail des jeunes mères. En fait, un certain renversement de perspective s'impose. La maternité dite précoce n'est peut-être pas un événement aussi incongru qu'il y paraît aux yeux de la société... et des intervenants sociaux en particulier.

\section{RÉFÉRENCES BIBLIOGRAPHIQUES}

Allen, I., et S. Bourke Dowling (1998). Teenage Mothers. Decisions and Outcomes, Londres, Royaume-Uni, Policy Studies Institute.

Charbonneau, J. (2003a). Adolescentes et mères. Histoires de maternité précoce et soutien du réseau social, Québec, Les Presses de l'Université Laval.

Charbonneau, J. (2003b). "La maternité adolescente au Québec. Une mise à l'épreuve des rapports entre la jeune mère et ses parents », dans E. MAUNAYE et M. Molgat (dir.), Les jeunes adultes et leurs parents. Autonomie, liens familiaux et modes de vie, Québec, Les Presses de l'Université Laval, p. 175-200.

ChARESt, D., et R. VILLEMURE (2002). Jeunes filles enceintes et mères adolescentes. Un portrait statistique, Québec, ministère de l'Éducation.

CLARK, S. (1999). "What do we know about unmarried mothers? », dans J. Wong et D. CheCKLAND (dir.), Teen Pregnancy and Parenting. Social and Ethical Issues, Toronto, University of Toronto Press.

ComBs-ORme, T. (1993). « Health effects of adolescent pregnancy: Implications for social work practice », Families in Society, vol. 74, p. 344-354.

COMITÉ CONSULTATIF FÉDÉRAL, PROVINCIAL ET TERRITORIAL SUR LA SANTÉ DE LA POPULATION (1999). Rapport statistique sur la santé de la population canadienne, Ottawa, Travaux publics et Services gouvernementaux Canada.

CONSEIL SUPÉRIEUR DE L'ÉDUCATION (1996). Contre l'abandon au secondaire. Rétablir l'appartenance scolaire, Avis à la ministre de l'Éducation, Québec, Conseil supérieur de l'éducation.

CÔTÉ, J. (1997). «Brève généalogie de discours sur les mères adolescentes. Le cas de l'administration québécoise de la santé publique », Anthropologie et Sociétés, vol. $21, \mathrm{n}^{\circ} 2$, p. 287-301. 
Côté, J., et A. Allahar (1994). Generation on Hold: Coming of Age in the Late Twentieth Century, Toronto, Stoddart.

COURNOYER, M. (1995). Grossesse, maternité et paternité à l'adolescence. Recueil de recension des écrits, Québec, INRS-Culture et Société / Institut québécois de recherche sur la culture.

DemaziÈRE, D., et C. Dubar (1994). L'insertion professionnelle des jeunes de bas niveau scolaire. Trajectoires biographiques et contextes structurels, document synthèse $n^{\circ} 91$, Marseille, Centre d'études et de recherches sur les qualifications, p. 71-85.

DÉVELOPPEMENT DES RESSOURCES HUMAINES CANADA (DRHC) (1998). Le secondaire, est-ce suffisant? Une analyse des résultats de l'Enquête de suivi auprès des sortants, 1995, Ottawa, Développement et ressources humaines Canada.

FÉDÉRATION DES CÉGEPS (1999). La réussite et la diplomation au collégial. Des chiffres et des engagements, Montréal.

Furstenberg, F.F., J. Brooks-Gunn et S.P. Morgan (1987). Adolescent Mothers in Later Life, New York, Cambridge University Press.

Gauthier, M., J. Hamel, M. Molgat, C. Trottier, C. Turcotte et M. Vultur (2004). L'insertion professionnelle et le rapport au travail des jeunes qui ont interrompu leurs études secondaires ou collégiales en 1996-1997. Étude rétrospective, Québec, INRS-Urbanisation, Culture et Société.

Hockaday, C., S. Crase, M.C. Shelley et D.F. Stockdale (2000). «A Prospective Study of Adolescent Pregnancy », Journal of Adolescence, vol. 23, p. 423-438.

HOFFMAN, S.D. (1998). « Teenage childbearing is not so bad after all... or is it? A review of the literature », Family Planning Perspectives, vol. 30, n 5, p. 236-240.

INSTITUT DE LA STATISTIQUE DU QUÉBEC (2004). Naissances et taux de fécondité selon le rang et le groupe d'âge de la mère, Québec, 1975-2003, Tableaux statistiques, [En ligne]. [www.stat.gouv.qc.ca/donstat/societe/demographie/naisn_deces/naissance/407.htm].

LE VAN, C. (1998). Les grossesses à l'adolescence. Normes sociales, réalités vécues, Paris, L'Harmattan.

MANSEAU, H., et M. Blais (2003). « Discours sur la grossesse et la sexualité : regards et actions féministes pour l'autonomie sexuelle et reproductive des adolescentes », dans Francine DescARRIES et Christine CORBEIL (dir.), Espace et temps de la maternité, Montréal, Les Éditions du remue-ménage, p. 215-238.

MAYNARD, R.A. (dir.) (1997). Kids Having Kids: The Costs and Social Consequences of Teenage Childbearing, Washington, DC, Urban Institute Press.

MINISTĖRE DE L'ÉDUCATION DU QUÉBEC (2003). Indicateurs de l'éducation, Québec, Gouvernement du Québec, [En ligne]. [http://www.meq.gouv.qc.ca/stat/indic03/indic03F/if2003.pdf].

Molgat, M., et E. MAUNAYE (2003). «L'acquisition de l'autonomie : enjeux et limites du rapport entre les jeunes et leurs parents », dans E. MAUNAYE et M. Molgat (dir.), Les jeunes adultes et leurs parents. Autonomie, liens familiaux et modes de vie, Québec, Les Presses de l'Université Laval, p. 1-25.

PAugam, S. (2000). Le salarié de la précarité, Paris, Presses universitaires de France. 
ROY, S., et D. CHAREST (2002). Organisation des services éducatifs offerts aux jeunes filles enceintes et aux mères adolescentes, Québec, Gouvernement du Québec, ministère de l'Éducation.

Vultur, M., C. Trottier et M. Gauthier (2002). « Les jeunes Québécois sans diplôme. Perspectives comparées sur l'insertion professionnelle et le rapport au travail », dans D.-G. TREMBLAY et L.-F. DAGENAIS (dir.), Ruptures, segmentations et mutations du marché du travail, Sainte-Foy, Presses de l'Université du Québec, p. 71-94. 